T.M. Rajendiran · M.T. Caudle $\cdot$ Martin L. Kirk

Ika Setyawati · Jeff W. Kampf · Vincent L. Pecoraro

\title{
Evaluating hydrogen bond interactions in enzymes containing Mn(III)-histidine complexation using manganese-imidazole complexes
}

Received: 8 July 2002 / Accepted: 1 October 2002 / Published online: 28 November 2002

(c) SBIC 2002

\begin{abstract}
It is often difficult to control hydrogen bond interactions in small molecule compounds that model metalloenzyme active sites. The imidazole-containing ligands 4,5-dicarboxyimidazole $\left(\mathrm{H}_{3} \mathrm{DCBI}\right)$ and 4,5-dicarboxy- $N$-methylimidazole $\left(\mathrm{H}_{2} \mathrm{MeDCBI}\right)$ allow examination of the effects of internal hydrogen bonding between carboxylate and imidazole nitrogen atoms. A new series of mononuclear manganese imidazole complexes have been prepared using these ligands: $\mathrm{Mn}(\mathrm{III})(\mathrm{salpn})\left(\mathrm{H}_{2} \mathrm{DCBI}\right)(\mathrm{DMF}) \quad$ (1), $\mathrm{Mn}(\mathrm{III})$ (salpn) (HMeDCBI) (2), Mn(III)(dtsalpn)(HMeDCBI) (3), [Mn(IV)(dtsalpn)(HMeDCBI)]PF 6 (4), $\mathrm{Mn}(\mathrm{III})$ (salpn) $\left(\mathrm{H}_{2} \mathrm{DCBI}\right)$ (5), $\mathrm{Mn}(\mathrm{III})(\mathrm{dtsalpn})\left(\mathrm{H}_{2} \mathrm{DCBI}\right) \quad(\mathbf{6})$, and $\mathrm{Mn}(\mathrm{IV})(\mathrm{dtsalpn})\left(\mathrm{H}_{2} \mathrm{DCBI}\right) \mathrm{PF}_{6}(\mathbf{8})$. Complexes 1, 2, 3, 5, and 6 have been prepared by direct reaction of salpn [salpn $=($ salicylideneaminato $)$-1,3-diaminopropane $)]$ or dtsalpn $\quad[\mathrm{dtsalpn}=(3,5$-di- $t$-butylsalicylideneaminato $)$ 1,3-diaminopropane)] and $\mathrm{H}_{3} \mathrm{DCBI}$ and $\mathrm{H}_{2} \mathrm{MeDCBI}$ with $\mathrm{Mn}(\mathrm{III})$ acetate, while complexes 4 and 8 were made by bulk electrolysis of complex $\mathbf{3}$ or $\mathbf{6}$ in dichloromethane. Complexes 1, 2, and $\mathbf{6}$ were characterized by X-ray diffraction. The impact of hydrogen bonding interactions of the complexes has been demonstrated by X-ray diffraction, cyclic voltammetry, and EPR spectroscopy. In all complexes the central metal ion is present in a six-coordinate geometry. Magnetic
\end{abstract}

T.M. Rajendiran · M.T. Caudle $\cdot$ I. Setyawati

J.W. Kampf · V.L. Pecoraro (ه)

Department of Chemistry, University of Michigan,

Ann Arbor, MI 48109-1055, USA

E-mail: vlpec@umich.edu

Tel.: + 1-734-7636148

Fax: + 1-734-6474865

M.L. Kirk

Department of Chemistry,

University of New Mexico, Albuquerque, NM 87131, USA

Permanent address: T.M. Rajendiran

Department of Chemistry,

Pondicherry University, R.V. Nagar, Kalapet,

Pondicherry 605 014, India susceptibility measurements confirm the spin and oxidation states of the complexes. The cyclic voltammograms of $\mathbf{3}$ and $\mathbf{6}$ in dichloromethane reveal single, reversible redox waves with $E_{1 / 2}=600 \mathrm{mV}$ and $690 \mathrm{mV}$, respectively. The X-band EPR spectrum of $\mathbf{4}$ shows a broad signal around $g=4.4$, and the corresponding complex $\mathbf{8}$ possesses a broad signal at slightly lower field $(g=5.5)$ than 4. These studies demonstrate that even small changes in the effective charge of the imidazole ligand can have a profound impact on the structure, spectroscopy, and magnetism of manganese(IV) complexes. We use these observations to present a model that may explain the origin of the $g=4.1$ signal in the $\mathrm{S}_{2}$ state of photosystem II.

Keywords Manganese enzymes ' Imidazole ·

Oxygen evolving complex

\section{Introduction}

The recognition that manganese is an important element in biology has increased dramatically over the past two decades. Originally, manganese was thought to serve simply as a divalent ion, similar to magnesium, in complexes with nucleotide di- and triphosphates. However, with the discovery that manganese is the essential component for water oxidation in the oxygen-evolving complex (OEC) $[1,2,3,4]$ and oxygen metabolism in human mitochondria via the manganese superoxide dismutase [5], this element became an important contributor to metalloenzymology. We now know that manganese can be found in a variety of enzymes as mononuclear $[6,7,8]$, binuclear $[9,10,11]$, or tetranuclear centers $[12,13]$. The relevant biological oxidation states for the metal are $\mathrm{Mn}(\mathrm{II}), \mathrm{Mn}(\mathrm{III})$, and $\mathrm{Mn}(\mathrm{IV})$. It is also possible that a formal $\mathrm{Mn}(\mathrm{V})$ oxidation state may be important in the OEC.

The OEC has multiple enzymatic oxidation levels that are termed $\mathrm{S}$ states. The most reduced form of the enzyme is $\mathrm{S}_{0}$ and the most oxidized form, capable of 
producing dioxygen, is $S_{4}$. While the $S_{0}, S_{1}$, and $S_{2}$ states have been probed using EPR spectroscopy, the $S_{2}$ state is the most complex of the five $\mathrm{S}$ states. Depending on the conditions that form $\mathrm{S}_{2}$, one can either observe a multiline signal centered at $g=2[4,14]$ or a broad, lowfield signal occurring at approximately $g=4[15,16]$. The multiline signal arises from an $S=1 / 2$ ground state of a mixed valent, tetranuclear Mn cluster with oxidation levels thought to be $\mathrm{Mn}(\mathrm{III}) \mathrm{Mn}(\mathrm{IV})_{3}$ [17]. The same nuclearity and oxidation states are thought to apply to the cluster that generates the $g=4.1$ signal; however, this center appears to have an $S=5 / 2$ ground state [18]. There are several ways to convert between conditions that form the multiline and $g=4.1$ signals. These methods include the temperature of illumination of the reaction center [19], the presence of small molecules such as ammonia [20] or fluoride [21, 22], and the presence of contaminating IR illumination [23, 24, 25, 26, 27, 28, 29]. Various explanations for the different signals have included causing a trapped protonation state of the cluster [30], physically changing the conformation of the cluster, or having valence/spin state isomers that are photoinduced [31, 32]. However, to date the precise explanation for these different signals is at best obscure.

In every structurally defined manganese enzyme $[6,7$, $8,9,10,11,12,13]$, imidazole serves as a donor to the metal. While the recent low-resolution X-ray structure [33] of photosystem II does not provide the identity of manganese ligands, spectroscopic studies have addressed this point. Using pulsed EPR techniques, Tang et al. [34] have shown that imidazole must be bound to at least some of the Mn ions in the OEC. These workers could not assess whether there were at least two different binding modes for unidentate imidazole coordination or whether a single imidizolate acted as a bridge between two Mn ions. Furthermore, X-ray studies have proven that imidazole binds to the manganese ions of serine/ threonine protein phosphatese-1 [35], arginase [36], catalase [9, 10], and superoxide dismutase [6]. In all of these cases the imidazole is bound as a unidentate ligand which can form hydrogen bonds to other protein residues.

Despite the ubiquitous presence of Mn-imidazole ligation, the number of model compounds that have imidazole as a ligand are relatively scarce $[37,38]$. We have recently employed the ligand 4,5-dicarboxyimidazole $\left(\mathrm{H}_{3} \mathrm{DCBI}\right)$, shown in Fig. 1, to form bi- and trinuclear manganese complexes. These compounds assess whether an imidazolate may serve as a bridging ligand between manganese ions in the OEC using a structural motif that is analogous to that seen in $\mathrm{Cu} / \mathrm{Zn}$ superoxide dismutase $[39,40]$. Through this imidazolate bridging motif, we were capable of preparing [(salpn) Mn(DCBI) Mn(sal$\mathrm{pn})]^{+}$which, due to the weak antiferromagnetic exchange coupling, was the first $\mathrm{Mn}(\mathrm{IV})_{2}$ complex that exhibited an X-band EPR spectrum [41]. In addition to containing two well-defined metal binding sites, the $\mathrm{H}_{3} \mathrm{DCBI}$ ligand also presents carboxylate groups that

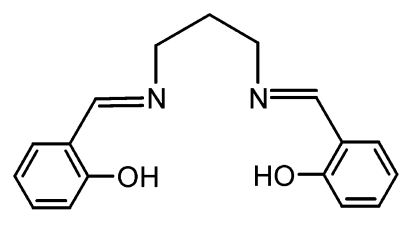

$\mathrm{H}_{2}$ salpn

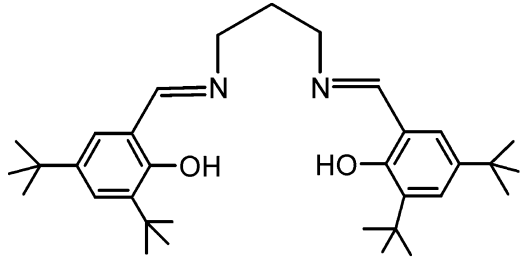

$\mathrm{H}_{2}$ dtsalpn<smiles>Cn1cnc(C(=O)O)c1C(=O)O</smiles>

$\mathrm{Me}-\mathrm{H}_{2} \mathrm{DCBI}$<smiles>O=C(O)c1nc[nH]c1C(=O)O</smiles>

$\mathrm{H}_{3} \mathrm{DCBI}$
Fig. 1 Ligands used in this study

may form internal hydrogen bonds to one another and also to the imidazole nitrogen atoms. Such an internal hydrogen bond network allows us to investigate the impact of hydrogen bonding on the structure and physical properties of manganese-imidazole species.

We report herein several new manganese-imidazole complexes in the +3 and +4 oxidation levels. In addition to having different oxidation states, these manganese complexes exhibit different carboxylate protonation states and may have the imidazole nitrogen alkylated. These different derivatives allow us to assess the structural changes that are associated with different extents of hydrogen bonding to the imidazole nitrogen. Based on these results, we will consider whether changes in the protonation state of the manganese cluster could be responsible for the different EPR spectral features of the $\mathrm{S}_{2}$ state of the OEC.

\section{Materials and methods}

All solvents used in these studies were obtained from Fischer in HPLC grade. Methylene chloride $\left(\mathrm{CH}_{2} \mathrm{Cl}_{2}\right)$ was distilled once over calcium hydride prior to use. Acetonitrile $(\mathrm{MeCN})$ was stirred over calcium hydride and anhydrous copper sulfate for $24 \mathrm{~h}$ and then distilled from phosphorus pentoxide before use. IR spectra were measured as $\mathrm{KBr}$ pellets on a Nicolet 5DX spectrometer. UV-visible spectra were measured on a Perkin-Elmer Lambda 9 spectrometer with a PC data station for acquisition. Temperaturedependent magnetic susceptibility data were measured using a Quantum Design 2000/2010 SQUID magnetometer and corrected for diamagnetic contributions using Pascal's constants for diamagnetic atoms and ions. X-band EPR spectra were measured on a Bruker ER-200E spectrometer with a $\mathrm{TE}_{102}$ cavity using a Varian liquid nitrogen cryostat to control temperature at $110 \mathrm{~K}$. Sub-77 K temperature control was maintained by an Oxford Instruments liquid helium cryostat. Electrochemistry data were collected on a BAS CV-27B potentiostat with chart recorder. Bulk electrolyses were performed using a BAS 100 electrochemical analyzer. The FAB mass spectra were recorded on a VG 70-250-S mass spectrometer/DATA systems using argon/xenon $(8 \mathrm{kV}, 1 \mathrm{~A})$ as the FAB gas and $m$-nitrobenzyl alcohol (NBA) as the matrix. It was equipped with the standard VG FAB ion source and an Ion Tech Saddle-field atom gun. 
Structure determination

Single-crystal X-ray diffraction experiments on 1 (see below) were performed on a Siemens P4 automated diffractometer equipped with a LT-2 low-temperature apparatus at $-75^{\circ} \mathrm{C}$ and graphite monochromatized $\mathrm{Mo} \mathrm{K}_{\alpha}$ radiation $(\lambda=0.71073 \AA$ ). Green blocklike crystals were grown from a solution of DMF/diethyl ether at room temperature. A crystal of dimensions $0.22 \times 0.20 \times 0.16 \mathrm{~mm}$ was lightly coated with Paratone- $\mathrm{N}$ oil and flash frozen on a glass fiber. Reflection indexing and cell constants are based on a matrix of 42 well-centered reflections in the range $10.2^{\circ} \geq 2 \theta \geq 24.9^{\circ}$. Additional details: scan method, $\theta / 2 \theta$; scan rate, variable $2-5 \mathrm{deg} / \mathrm{min}$; background to scan ratio, $0.5 ; 2 \theta$ scan range, $5-52(h:-1 / 12 ; k:-1 / 11 ; 1:-31 / 31) ; 6234$ reflections measured; merged to 4705 unique reflections; $R_{\text {int }}=0.0320$; no correction for absorption. The structure was solved by direct methods and 427 parameters were refined in a full matrix with the program SHELXTL PLUS [42]. All non-hydrogen atoms were allowed to refine anisotropically with hydrogen atoms placed in idealized positions or allowed to refine isotropically. Neutral atom scattering factors and corrections for anomalous dispersion were taken from published data [43]. Table 1 contains further crystallographic details.

Single-crystal X-ray diffraction experiments on 2 (see below) were performed on a Syntex P2(1) automated diffractometer at $23{ }^{\circ} \mathrm{C}$ with graphite monochromatized Mo $\mathrm{K}_{\alpha}$ radiation $(\lambda=0.71073 \AA)$. Green block-like crystals were grown from a solution of $\mathrm{CH}_{2} \mathrm{Cl}_{2}$ /toluene at room temperature. A crystal of approximate dimensions $0.52 \times 0.48 \times 0.26 \mathrm{~mm}$ was lightly coated with epoxy and mounted on a glass fiber. Reflection indexing and cell constants are based on a matrix of 40 well-centered reflections in the range $10.0^{\circ}=2 \theta \geq 24.8^{\circ}$. Additional details: scan method, $\theta / 2 \theta$; scan rate variable, $2-5 \mathrm{deg} / \mathrm{min}$; background to scan ratio, $0.5 ; 2 \theta$ scan range, $5-52^{\circ}(h: 0 / 14 ; k: 0 / 13 ; 1:-26 / 25) ; 5425$ reflections measured; merged to 5168 unique reflections; $R_{\text {int }}=0.0238$; correction for absorption by psi scans. The structure was solved by direct methods and 391 parameters were refined in a full matrix with the SHELXTL PLUS program.
Green plates of $\mathbf{6}$ were crystallized from an acetonitrile solution at $-20{ }^{\circ} \mathrm{C}$. A crystal of dimensions $0.22 \times 0.16 \times 0.08 \mathrm{~mm}$ was mounted on a standard Bruker SMART CCD X-ray diffractometer equipped with a normal focus Mo-target X-ray tube $(\lambda=0.71071 \AA)$ operated at $2000 \mathrm{~W}$ power $(50 \mathrm{kV}, 40 \mathrm{~mA})$ and LT-2 low-temperature device. The X-ray intensities were measured at $158(2) \mathrm{K}$; the detector was placed at a distance of $4.939 \mathrm{~cm}$ from the crystal. A total of 2172 frames were collected with a scan width of $0.3^{\circ}$ in $\omega$ and $\phi$ with an exposure time of $30 \mathrm{~s} /$ frame. The frames were integrated with the Bruker SAINT software package with a narrow frame algorithm. The integration of the data yielded a total of 123,142 reflections to a maximum $2 \theta$ value of $52.85^{\circ}$, of which 22,767 were unique data. Owing to the lack of high-angle observed data from the weakly diffracting crystal, resolution of the refinement was limited to $1.08 \AA$ during the refinement, leaving 10,707 unique data and 7146 greater that $2 \sigma(I)$. The final cell constants (Table 2) were based on the $x y z$ centroids of 6025 reflections above $102 \sigma(I)$. Analysis of the data showed negligible decay during data collection; the data were processed with SADABS and corrected for absorption. The structure was solved and refined with the Bruker SHELXTL (version 5.10) software package, using the space group $P 2 / c$ with $Z=4$ for the formula $\mathrm{C}_{116} \mathrm{H}_{159.5} \mathrm{Mn}_{3} \mathrm{~N}_{13.5} \mathrm{O}_{14.5}$, which includes the contribution of lattice solvate molecules of $\mathrm{MeCN}$ and a partial occupancy water molecule. All non-hydrogen atoms were refined anisotropically with the hydrogen atoms located on a difference Fourier map and allowed to refine isotropically or placed in idealized positions. Full-matrix least-squares refinement based on $F^{2}$ converged at $R 1=0.0811$ and $w R 2=0.2298$ [based on $I>2 \sigma(I)$ ], $R 1=0.1165$ and $w R 2=0.2537$ for all data. Selected bond lengths and angles of 1, 2, and 6 are given in Table 2.

\section{Synthesis of ligands}

4,5-Dicyanoimidazole was obtained from Aldrich and hydrolyzed by refluxing in $1.0 \mathrm{M}$ aqueous $\mathrm{HCl}$ to yield 4,5-dicarboxyimidazole $\left(\mathrm{H}_{3} \mathrm{DCBI}\right)$ [44]. Analysis: found (calc): C, 62.4 (62.4); H, 9.8 (10.0);

Table 1 Crystallographic data for Mn(salpn)(H $\left.\mathrm{H}_{2} \mathrm{DCBI}\right)(\mathrm{DMF})(\mathbf{1}), \mathrm{Mn}(\mathrm{III})(\mathrm{salpn})(\mathrm{HMeDCBI})(\mathbf{2})$, and $\mathrm{Mn}(\mathrm{dtsalpn})\left(\mathrm{H}_{2} \mathrm{DCBI}\right)(\mathbf{6})$

\begin{tabular}{|c|c|c|c|}
\hline & 1 & 2 & 6 \\
\hline Empirical formula & $\mathrm{C}_{25} \mathrm{H}_{28} \mathrm{MnN}_{5} \mathrm{O}_{7}$ & $\mathrm{C}_{23} \mathrm{H}_{21} \mathrm{MnN}_{4} \mathrm{O}_{6}$ & $\mathrm{C}_{116} \mathrm{H}_{159.5} \mathrm{Mn}_{3} \mathrm{~N}_{13.5} \mathrm{O}_{14.5}$ \\
\hline Formula weight & 565.46 & 504.38 & 2139.89 \\
\hline Temperature (K) & $178(2)$ & 293(2) & $158(2)$ \\
\hline Wavelength $(\AA)$ & 0.71073 & 0.71073 & 0.71073 \\
\hline Crystal system & Monoclinic & Monoclinic & Monoclinic \\
\hline Space group & $P 2(1) / n$ & $P 2(1) / n$ & $P 2 / c$ \\
\hline$a(\AA)$ & $9.8140(10)$ & $11.142(3)$ & $32.062(3)$ \\
\hline$b(\AA)$ & $9.7180(10)$ & $10.129(2)$ & $13.4330(12)$ \\
\hline$c(\mathrm{~A})$ & $25.783(2)$ & $20.354(5)$ & $32.232(3)$ \\
\hline$\alpha\left({ }^{\circ}\right)$ & 90 & 90 & 90 \\
\hline$\beta\left(^{\circ}\right)$ & $100.600(10)$ & $102.18(2)$ & $111.8690(10)$ \\
\hline$\gamma\left({ }^{\circ}\right)$ & 90 & 90 & 90 \\
\hline Volume, $Z\left(\AA^{3}\right)$ & $2417.0(4)$ & $2245.4(9)$ & $12,883.3(19)$ \\
\hline Density (calc) $\left(\mathrm{Mg} / \mathrm{m}^{3}\right)$ & 1.554 & 1.492 & 1.103 \\
\hline Absorption coefficient $\left(\mathrm{mm}^{-1}\right)$ & 0.604 & 0.636 & 0.349 \\
\hline$F(000)$ & 1176 & 1040 & 4564 \\
\hline Crystal size $(\mathrm{mm})$ & $0.22 \times 0.20 \times 0.16$ & $0.52 \times 0.48 \times 0.26$ & $0.08 \times 0.16 \times 0.22$ \\
\hline$\theta$ range $\left(^{\circ}\right)$ & 2.64 to 26.01 & 2.75 to 27.56 & 1.36 to 19.21 \\
\hline Reflns collected & 6234 & 5425 & 123,142 \\
\hline Independent reflns & $4705[R(\mathrm{int})=0.0320]$ & $5168[R(\mathrm{int})=0.0238]$ & $10,707[R(\mathrm{int})=0.0505]$ \\
\hline Absorption correction & None & Psi scans & Semi-empirical from equivalents \\
\hline Data/restraints/parameters & $4697 / 0 / 434$ & $5078 / 0 / 391$ & $10707 / 6 / 1306$ \\
\hline Goodness-of-fit on $F^{2}$ & 0.985 & 1.031 & 1.044 \\
\hline Final $R$ indices $[I>2 \sigma(I)]$ & $R 1=0.0479$ & $R 1=0.0367$ & $R 1=0.0811$ \\
\hline & $w R 2=0.1216$ & $w R 2=0.0860$ & $w R 2=0.2298$ \\
\hline$R$ indices (all data) & $R 1=0.0688$ & $R 1=0.0533$ & $R 1=0.1165$ \\
\hline Largest diff. peak and hole $\left(\mathrm{e} \AA^{-3}\right)$ & $\begin{array}{l}w R 2=0.1283 \\
0.772 \text { and }-0.618\end{array}$ & $w R 2=0.1001$ & $w R 2=0.2537$ \\
\hline & & 0.205 dild -0.290 & \\
\hline
\end{tabular}


Table 2 Selected bond lengths $(\AA)$ and angles $\left({ }^{\circ}\right)$ for $\mathrm{Mn}(\mathrm{sal}-$ pn)( $\left.\mathrm{H}_{2} \mathrm{DCBI}\right)(\mathrm{DMF})$ (1), $\mathrm{Mn}(\mathrm{III})(\mathrm{salpn})(\mathrm{HMeDCBI})$ (2), and $\mathrm{Mn}(\mathrm{dtsalpn})\left(\mathrm{H}_{2} \mathrm{DCBI}\right)(\mathbf{6})^{\mathrm{a}}$

\begin{tabular}{|c|c|}
\hline \multicolumn{2}{|c|}{$\mathrm{Mn}(\mathrm{salpn})\left(\mathrm{H}_{2} \mathrm{DCBI}\right)(\mathrm{DMF})(\mathbf{1})$} \\
\hline $\mathrm{Mn}(1)-\mathrm{O}(1)$ & $1.877(2)$ \\
\hline $\mathrm{Mn}(1)-\mathrm{O}(2)$ & $1.891(2)$ \\
\hline $\mathrm{Mn}(1)-\mathrm{N}(2)$ & $2.029(3)$ \\
\hline $\mathrm{Mn}(1)-\mathrm{N}(1)$ & $2.037(3)$ \\
\hline $\operatorname{Mn}(1)-O(7)$ & $2.241(2)$ \\
\hline $\mathrm{Mn}(1)-\mathrm{O}(5)$ & $2.291(2)$ \\
\hline $\mathrm{O}(1)-\mathrm{Mn}(1)-\mathrm{O}(2)$ & $83.66(9)$ \\
\hline $\mathrm{O}(1)-\mathrm{Mn}(1)-\mathrm{N}(2)$ & $172.98(11)$ \\
\hline $\mathrm{O}(2)-\mathrm{Mn}(1)-\mathrm{N}(2)$ & $90.02(11)$ \\
\hline $\mathrm{O}(1)-\mathrm{Mn}(1)-\mathrm{N}(1)$ & $90.59(10)$ \\
\hline $\mathrm{O}(2)-\mathrm{Mn}(1)-\mathrm{N}(1)$ & $174.24(10)$ \\
\hline $\mathrm{N}(2)-\mathrm{Mn}(1)-\mathrm{N}(1)$ & $95.74(12)$ \\
\hline $\mathrm{O}(1)-\mathrm{Mn}(1)-\mathrm{O}(7)$ & $95.38(9)$ \\
\hline $\mathrm{O}(2)-\mathrm{Mn}(1)-\mathrm{O}(7)$ & $96.06(9)$ \\
\hline $\mathrm{N}(2)-\mathrm{Mn}(1)-\mathrm{O}(7)$ & $82.19(11)$ \\
\hline $\mathrm{N}(1)-\mathrm{Mn}(1)-\mathrm{O}(7)$ & $84.86(9)$ \\
\hline $\mathrm{O}(1)-\mathrm{Mn}(1)-\mathrm{O}(5)$ & $97.03(9)$ \\
\hline $\mathrm{O}(2)-\mathrm{Mn}(1)-\mathrm{O}(5)$ & $85.90(9)$ \\
\hline $\mathrm{N}(2)-\mathrm{Mn}(1)-\mathrm{O}(5)$ & $85.54(11)$ \\
\hline $\mathrm{N}(1)-\mathrm{Mn}(1)-\mathrm{O}(5)$ & $94.42(9)$ \\
\hline $\mathrm{O}(7)-\mathrm{Mn}(1)-\mathrm{O}(5)$ & $167.57(8)$ \\
\hline $\mathrm{C}(1)-\mathrm{O}(1)-\mathrm{Mn}(1)$ & $132.6(2)$ \\
\hline $\mathrm{C}(17)-\mathrm{O}(2)-\mathrm{Mn}(1)$ & $133.0(2)$ \\
\hline $\mathrm{C}(22)-\mathrm{O}(5)-\mathrm{Mn}(1)$ & $129.9(2)$ \\
\hline $\mathrm{C}(23)-\mathrm{O}(7)-\mathrm{Mn}(1)$ & $131.1(2)$ \\
\hline $\mathrm{C}(7)-\mathrm{N}(1)-\mathrm{Mn}(1)$ & $123.4(2)$ \\
\hline $\mathrm{C}(8)-\mathrm{N}(1)-\mathrm{Mn}(1)$ & $123.1(2)$ \\
\hline \multicolumn{2}{|c|}{ Mn(III)(salpn)(HMeDCBI) (2) } \\
\hline $\mathrm{Mn}(1)-\mathrm{O}(1)$ & $1.8628(14)$ \\
\hline $\mathrm{Mn}(1)-\mathrm{O}(2)$ & $1.956(2)$ \\
\hline $\operatorname{Mn}(1)-N(2)$ & $1.995(2)$ \\
\hline $\operatorname{Mn}(1)-N(1)$ & $2.111(2)$ \\
\hline $\mathrm{Mn}(1)-\mathrm{O}(3)$ & $2.162(2)$ \\
\hline $\operatorname{Mn}(1)-N(3)$ & $2.245(2)$ \\
\hline $\mathrm{O}(1)-\mathrm{Mn}(1)-\mathrm{O}(2)$ & $91.43(7)$ \\
\hline $\mathrm{O}(1)-\mathrm{Mn}(1)-\mathrm{N}(2)$ & $177.49(6)$ \\
\hline $\mathrm{O}(2)-\mathrm{Mn}(1)-\mathrm{N}(2)$ & $89.37(7)$ \\
\hline $\mathrm{O}(1)-\mathrm{Mn}(1)-\mathrm{N}(1)$ & $88.55(7)$ \\
\hline $\mathrm{O}(2)-\mathrm{Mn}(1)-\mathrm{N}(1)$ & $110.63(6)$ \\
\hline $\mathrm{N}(2)-\mathrm{Mn}(1)-\mathrm{N}(1)$ & $88.94(7)$ \\
\hline $\mathrm{O}(1)-\mathrm{Mn}(1)-\mathrm{O}(3)$ & $93.02(7)$ \\
\hline $\mathrm{O}(2)-\mathrm{Mn}(1)-\mathrm{O}(3)$ & $163.83(6)$ \\
\hline $\mathrm{N}(2)-\mathrm{Mn}(1)-\mathrm{O}(3)$ & $86.85(7)$ \\
\hline $\mathrm{N}(1)-\mathrm{Mn}(1)-\mathrm{O}(3)$ & $85.03(6)$ \\
\hline $\mathrm{O}(1)-\mathrm{Mn}(1)-\mathrm{N}(3)$ & $89.46(6)$ \\
\hline $\mathrm{O}(2)-\mathrm{Mn}(1)-\mathrm{N}(3)$ & $90.04(6)$ \\
\hline $\mathrm{N}(2)-\mathrm{Mn}(1)-\mathrm{N}(3)$ & $92.92(7)$ \\
\hline $\mathrm{N}(1)-\mathrm{Mn}(1)-\mathrm{N}(3)$ & $159.28(6)$ \\
\hline $\mathrm{O}(3)-\mathrm{Mn}(1)-\mathrm{N}(3)$ & $74.48(6)$ \\
\hline $\mathrm{C}(1)-\mathrm{O}(1)-\mathrm{Mn}(1)$ & $125.22(13)$ \\
\hline $\mathrm{C}(17)-\mathrm{O}(2)-\mathrm{Mn}(1)$ & $125.39(13)$ \\
\hline $\mathrm{C}(21)-\mathrm{O}(3)-\mathrm{Mn}(1)$ & $119.64(13)$ \\
\hline $\mathrm{C}(7)-\mathrm{N}(1)-\mathrm{Mn}(1)$ & $120.0(2)$ \\
\hline $\mathrm{C}(8)-\mathrm{N}(1)-\mathrm{Mn}(1)$ & $122.36(14)$ \\
\hline $\mathrm{C}(11)-\mathrm{N}(2)-\mathrm{Mn}(1)$ & $123.4(2)$ \\
\hline $\mathrm{C}(10)-\mathrm{N}(2)-\mathrm{Mn}(1)$ & $119.56(14)$ \\
\hline $\mathrm{C}(18)-\mathrm{N}(3)-\mathrm{Mn}(1)$ & $141.38(14)$ \\
\hline $\mathrm{C}(20)-\mathrm{N}(3)-\mathrm{Mn}(1)$ & $111.92(13)$ \\
\hline \multicolumn{2}{|c|}{$\mathrm{Mn}($ dtsalpn $)\left(\mathrm{H}_{2} \mathrm{DCBI}\right)$} \\
\hline $\mathrm{Mn}(3)-\mathrm{O}(9)$ & $1.858(6)$ \\
\hline $\operatorname{Mn}(3)-N(8)$ & $1.974(8)$ \\
\hline $\mathrm{Mn}(3)-\mathrm{O}(10)$ & $2.001(6)$ \\
\hline $\operatorname{Mn}(3)-N(7)$ & $2.013(8)$ \\
\hline $\operatorname{Mn}(3)-N(9)$ & $2.159(8)$ \\
\hline $\mathrm{Mn}(3)-\mathrm{O}(11)$ & $2.265(7)$ \\
\hline $\mathrm{O}(9)-\mathrm{Mn}(3)-\mathrm{N}(8)$ & $177.4(3)$ \\
\hline
\end{tabular}

Table 2 (Contd.)

\begin{tabular}{ll}
$\mathrm{O}(9)-\mathrm{Mn}(3)-\mathrm{O}(10)$ & $92.1(3)$ \\
$\mathrm{N}(8)-\mathrm{Mn}(3)-\mathrm{O}(10)$ & $88.3(3)$ \\
$\mathrm{O}(9)-\mathrm{Mn}(3)-\mathrm{N}(7)$ & $90.6(3)$ \\
$\mathrm{N}(8)-\mathrm{Mn}(3)-\mathrm{N}(7)$ & $86.8(3)$ \\
$\mathrm{O}(10)-\mathrm{Mn}(3)-\mathrm{N}(7)$ & $108.1(3)$ \\
$\mathrm{O}(9)-\mathrm{Mn}(3)-\mathrm{N}(9)$ & $88.2(3)$ \\
$\mathrm{N}(8)-\mathrm{Mn}(3)-\mathrm{N}(9)$ & $94.4(3)$ \\
$\mathrm{O}(10)-\mathrm{Mn}(3)-\mathrm{N}(9)$ & $89.0(3)$ \\
$\mathrm{N}(7)-\mathrm{Mn}(3)-\mathrm{N}(9)$ & $162.8(3)$ \\
$\mathrm{O}(9)-\mathrm{Mn}(3)-\mathrm{O}(11)$ & $92.7(2)$ \\
$\mathrm{N}(8)-\mathrm{Mn}(3)-\mathrm{O}(11)$ & $87.6(3)$ \\
$\mathrm{O}(10)-\mathrm{Mn}(3)-\mathrm{O}(11)$ & $162.9(3)$ \\
$\mathrm{N}(7)-\mathrm{Mn}(3)-\mathrm{O}(11)$ & $88.3(3)$ \\
$\mathrm{N}(9)-\mathrm{Mn}(3)-\mathrm{O}(11)$ & $74.7(3)$ \\
$\mathrm{C}(74)-\mathrm{O}(9)-\mathrm{Mn}(3)$ & $130.0(6)$ \\
$\mathrm{C}(92)-\mathrm{O}(10)-\mathrm{Mn}(3)$ & $131.9(6)$ \\
$\mathrm{C}(107)-\mathrm{O}(11)-\mathrm{Mn}(3)$ & $115.1(7)$ \\
$\mathrm{C}(72)-\mathrm{N}(7)-\mathrm{Mn}(3)$ & $123.4(7)$ \\
$\mathrm{C}(87)-\mathrm{N}(7)-\mathrm{Mn}(3)$ & $118.4(6)$ \\
$\mathrm{C}(90)-\mathrm{N}(8)-\mathrm{Mn}(3)$ & $125.5(7)$ \\
$\mathrm{C}(89)-\mathrm{N}(8)-\mathrm{Mn}(3)$ & $118.2(6)$ \\
$\mathrm{C}(105)-\mathrm{N}(9)-\mathrm{Mn}(3)$ & $138.4(7)$ \\
$\mathrm{C}(106)-\mathrm{N}(9)-\mathrm{Mn}(3)$ & $114.3(7)$ \\
\hline
\end{tabular}

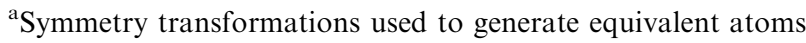

$\mathrm{N}, 10.4$ (10.2). 4,5-Dicarboxy-3-methylimidazole $\left(\mathrm{H}_{2} \mathrm{MeDCBI}\right)$ was synthesized as reported in the literature [44].

\section{$\mathrm{Mn}^{\mathrm{III}}$ (salpn)(DMF)(H $\left.{ }_{2} \mathrm{DCBI}\right)(\mathbf{1})$}

$\mathrm{Mn}^{\mathrm{III}}$ (salpn)(acac) was prepared and analyzed as described previously [45]. $\mathrm{Mn}^{\mathrm{III}}$ (salpn)(acac) $(2.0 \mathrm{~g}, 4.6 \mathrm{mmol})$ and $\mathrm{H}_{3} \mathrm{DCBI}$ $(0.72 \mathrm{~g}, 4.6 \mathrm{mmol})$ were allowed to react in $100 \mathrm{~mL} \mathrm{MeCN}$. After $10 \mathrm{~h}, 2.3 \mathrm{~g}$ of a green, very insoluble product was formed. This bright-green solid was dissolved in degassed pyridine and a large excess of $\mathrm{Et}_{2} \mathrm{O}$ added. Filtration under nitrogen produced a green hygroscopic powder. Dissolution of this powder in $\mathrm{MeCN}$ and allowing the solution to evaporate slowly gave a green crystalline precipitate that could not be redissolved in $\mathrm{MeCN}$. Crystallization by slow vapor diffusion of $\mathrm{Et}_{2} \mathrm{O}$ into a solution of $\mathbf{1}$ in DMF gave a new complex, which did not readily lose the DMF solvate upon drying in vacuo. Analysis: found (calc): C, 53.4 (53.3); H, 4.8 (4.7); N, 12.7 (12.4); Mn, 10.5 (9.8).

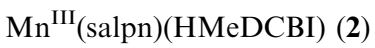

$\mathrm{Mn}^{\mathrm{III}}$ (salpn)(acac) $(4.0 \mathrm{~g}, 9.2 \mathrm{mmol})$ was dissolved in $\mathrm{MeCN}$ (150 mL) and allowed to react with $\mathrm{H}_{2} \mathrm{MeDCBI}(1.6 \mathrm{~g}, 9.2 \mathrm{mmol})$. After stirring overnight, $4.6 \mathrm{~g}(100 \%)$ of a green solid had precipitated and was collected. Recrystallization of this solid from acetone gave pure $\mathrm{Mn}^{\mathrm{III}}$ (salpn) $\mathrm{H}(\mathrm{MeDCBI}) \cdot(\text { acetone })_{0.5}$, 1.(acetone $)_{0.5}$. Analysis: found (calc): C, 54.7 (54.8); H, 4.7 (4.5); N, 10.6 (10.5); Mn, 10.6 (10.3). IR: $v_{\mathrm{C}=\mathrm{O}}=1710, v_{\mathrm{C}=\mathrm{N}}=1614 \mathrm{~cm}^{-1}$. Negative ion FAB-MS: $[1 \cdot(\text { acetone })]^{-}, 562 ;[1]^{-}, 504$.

\section{$\mathrm{Mn}^{\mathrm{III}}$ (dtbsalpn)(HMeDCBI) (3)}

1,3-Diaminopropane (1.6 g, $21 \mathrm{mmol})$ and 3,5-di-tert-butyl-2hydroxysalicylaldehyde $(10 \mathrm{~g}, 43 \mathrm{mmol})$ were reacted together in $\mathrm{MeOH}(200 \mathrm{~mL})$, yielding a bright yellow suspension. $\mathrm{Mn}^{\mathrm{III}}(\mathrm{OAc})_{3} \cdot 2 \mathrm{H}_{2} \mathrm{O}(5.7 \mathrm{~g}, 21 \mathrm{mmol})$ was added and the mixture gently refluxed for $24 \mathrm{~h}$ to give a dark green precipitate. This solid was collected by filtration and redissolved in $\mathrm{MeOH}(200 \mathrm{~mL})$ with aqueous $\mathrm{HClO}_{4}$ (1 equiv). A 10 -fold excess of $\mathrm{KPF}_{6}$ was dissolved in water $(100 \mathrm{~mL})$ and added to the $\mathrm{MeOH}$ solution slowly to give a brown precipitate. This solid was collected and recrystallized from 
water $/ \mathrm{MeOH}$ to give a $93 \%$ overall yield of $\mathrm{Mn}^{\mathrm{III}}$ (dtbsalpn) $\left(\mathrm{H}_{2} \mathrm{O}\right)_{2}{ }^{+} \mathrm{PF}_{6}{ }^{-}$. Analysis: found (calc): $\mathrm{C}, 53.6$ (53.5); H, 7.1 (7.1); N, 4.0 (3.8); Mn, 7.0 (7.4). IR: $v(\mathrm{C}=\mathrm{N}), 1614 ; v\left(\mathrm{PF}_{6}^{-}\right), 845 \mathrm{~cm}^{-1}$.

$\mathrm{Mn}^{\mathrm{III}}$ (dtbsalpn) $\left(\mathrm{H}_{2} \mathrm{O}\right)_{2}{ }^{+} \mathrm{PF}_{6}{ }^{-}$could also be synthesized by adding aqueous $\mathrm{HPF}_{6}$ (1 equiv) to $\mathrm{Mn}(\mathrm{dtbsalpn})\left(\mathrm{MeCO}_{2}\right)$ in $\mathrm{MeOH}$ and stirring for $2 \mathrm{~h}$ at room temperature. To this mixture, water was added until all the brown solid had been precipitated. The yield of the resulting dark brown solid ([Mn(dtbsalpn) $\left.\left.\left(\mathrm{H}_{2} \mathrm{O}\right)_{2}\right] \mathrm{PF}_{6}\right)$ was $93 \%$. IR spectroscopy and elemental analysis confirmed the correct formulation.

$\mathrm{H}_{2} \mathrm{MeDCBI}(0.46 \mathrm{~g}, 2.7 \mathrm{mmol})$ and $\mathrm{Et}_{4} \mathrm{NOAc}(0.71 \mathrm{~g}$, $2.7 \mathrm{mmol})$ were dissolved in $\mathrm{MeCN}(150 \mathrm{~mL}) . \mathrm{Mn}^{\text {III }}$ (dtbsalpn) $\left(\mathrm{H}_{2} \mathrm{O}\right)_{2}{ }^{+} \mathrm{PF}_{6}^{-}(2.0 \mathrm{~g}, 2.7 \mathrm{mmol})$ was added and the reaction stirred overnight to yield a green-brown solution. The solvent was removed by rotary evaporation and the solid residue extracted with toluene. After filtration, the toluene solvent was removed by rotary evaporation to yield $0.80 \mathrm{~g}(40 \%)$ of $\mathrm{Mn}^{\text {III }}$ (dtbsalpn)H(MeD$\mathrm{CBI}$ )(toluene $)_{0.5}, \mathbf{2}$ (toluene $)_{0.5}$. Analysis: found (calc): C, 65.5 (65.9); H, 7.2 (7.4); N, 7.1 (7.2); Mn, 7.0 (7.1). IR: $v(\mathrm{C}=\mathrm{N}), 1611$; $v(\mathrm{C}=\mathrm{O}), 1715 \mathrm{~cm}^{-1} \cdot \mu_{\mathrm{eff}}(300 \mathrm{~K})=4.9 \mathrm{BM}$

\section{$\left[\mathrm{Mn}^{\mathrm{IV}}(\mathrm{dtbsalpn})(\mathrm{HMeDCBI})\right] \mathrm{PF}_{6}(\mathbf{4})$}

Complex $2(0.44 \mathrm{~g}, 0.60 \mathrm{mmol})$ was dissolved in $\mathrm{CH}_{2} \mathrm{Cl}_{2}(130 \mathrm{~mL})$ containing $0.10 \mathrm{M} \mathrm{TBAPF}_{6}$. This solution was bulk electrolyzed on a platinum mesh at $900 \mathrm{mV}$ against a silver wire reference placed directly in the analyte solution. The platinum counter electrode was placed in $0.10 \mathrm{M} \mathrm{TBA}^{+} \mathrm{PF}_{6}{ }^{-}$in $\mathrm{CH}_{2} \mathrm{Cl}_{2}$ and isolated from the bulk solution by a fine glass frit $(1.5 \mathrm{~cm}$ in diameter). After passage of $61 \mathrm{C}$, the solution had turned a dark forest-green color. The solvent was removed by evaporation under a stream of dry nitrogen. The resulting solid residue was extracted with a mixture of $\mathrm{Et}_{2} \mathrm{O}$ / $\mathrm{CH}_{2} \mathrm{Cl}_{2}$ (10:1) and filtered until the remaining solid residue was white. The filtrate was evaporated and the dark green solid washed with $\mathrm{Et}_{2} \mathrm{O}$ and hexane to give $0.44 \mathrm{~g}(83 \%)$ of $\left[\mathrm{Mn}^{\mathrm{IV}}(\mathrm{dtb}-\right.$ salpn)(HMeDCBI)] $\mathrm{PF}_{6} \cdot 0.7 \mathrm{C}_{16} \mathrm{H}_{36} \mathrm{NPF}_{6}\left(3 \cdot 0.7 \mathrm{TBAPF}_{6}\right)$. Analysis: found (calc): C, $53.0(52.7) ; \mathrm{H}, 7.2(6.9) ; \mathrm{N}, 5.7(5.8)$. IR: $v(\mathrm{C}=\mathrm{O})$, $1748 ; v(\mathrm{C}=\mathrm{N}), 1603 ; v\left(\mathrm{PF}_{6}\right), 845 \mathrm{~cm}^{-1} . \mu_{\mathrm{eff}}(300 \mathrm{~K})=3.9 \mathrm{BM}$.

\section{$\mathrm{Mn}^{\mathrm{III}}$ (salpn) $\mathrm{H}_{2} \mathrm{DCBI}$ (5)}

$\mathrm{Mn}^{\mathrm{III}}$ (salpn)(acac) $(2.0 \mathrm{~g}, 4.6 \mathrm{mmol})$ and $\mathrm{H}_{3} \mathrm{DCBI} \quad(0.72 \mathrm{~g}$, $4.6 \mathrm{mmol})$ were reacted in $\mathrm{MeCN}(100 \mathrm{~mL})$. After $10 \mathrm{~h}, 2.3 \mathrm{~g}$ of a green, very insoluble product was formed. This bright-green solid was dissolved in degassed pyridine and a large excess of $\mathrm{Et}_{2} \mathrm{O}$ added. Filtration under nitrogen produced a green hygroscopic powder. Dissolution of this powder in $\mathrm{MeCN}$ and allowing the solution to evaporate slowly gave a green crystalline precipitate that could not be redissolved in $\mathrm{MeCN}$. Analysis of this complex was consistent with $\mathrm{Mn}^{\mathrm{III}}$ (salpn) $\mathrm{H}_{2}$ DCBI (5). Analysis: found (calc): C, 53.6 (53.9); H, 4.0 (3.9); N, 11.6 (11.5); Mn, 10.9 (11.2).

$\mathrm{Mn}(\mathrm{III})(\mathrm{dtbsalpn})\left(\mathrm{H}_{2} \mathrm{DCBI}\right)(6)$ and $\mathrm{Mn}_{2}(\mathrm{III})(\mathrm{dtbsalpn})_{2}$ (HDCBI) (7)

[Mn(dtbsalpn $\left.)\left(\mathrm{H}_{2} \mathrm{O}\right)_{2}\right] \mathrm{PF}_{6}(188 \mathrm{mg}, 0.25 \mathrm{mmol})$ was dissolved in distilled $\mathrm{MeCN}(30 \mathrm{~mL})$ and placed in a dropping funnel. This solution was added dropwise into a slurry of $\mathrm{H}_{3}$ DCBI $(48 \mathrm{mg}$, $0.31 \mathrm{mmol})$ in $\mathrm{MeCN} / \mathrm{MeOH}(15 \mathrm{~mL} / 35 \mathrm{~mL})$ over a period of $1.5 \mathrm{~h}$. When 0.5 equiv of $\left[\mathrm{Mn}(\mathrm{dtbsalpn})\left(\mathrm{H}_{2} \mathrm{O}\right)_{2}\right] \mathrm{PF}_{6}$ had been added, $1.0 \mathrm{M}$ TBAOH $(0.10 \mathrm{~mL})$ was added. When the addition of $\mathrm{Mn}$ starting material had been completed, further TBAOH $(0.15 \mathrm{~mL})$ was added. The mixture was stirred at room temperature for another $15 \mathrm{~min}$. The undissolved white solid was removed by filtration, and the greenish brown filtrate was collected. The solvent was removed in vacuo, leaving a dark green solid. A minimal amount of $\mathrm{MeCN}$ was added, and this slurry was stored at $-20{ }^{\circ} \mathrm{C}$ overnight. A greenish white solid was filtered and discarded. The remaining filtrate was left at room temperature for slow evapora- tion (not longer than one day), yielding $\mathrm{Mn}(\mathrm{dtbsalpn})\left(\mathrm{H}_{2} \mathrm{DCBI}\right)$ (92 $\mathrm{mg}, 51 \%$ ), which was filtered as a dark green solid. More green solid $(30 \mathrm{mg})$ was isolated from the remaining filtrate, bringing the total yield to $68 \%$. Analysis: found (calc): C, 63.5 (63.8); H, 7.3 (7.2); N, 7.9 (7.8). IR: $v(\mathrm{C}=\mathrm{N})$ 1611, $v(\mathrm{~N}-\mathrm{H}) 3152, v(\mathrm{C}=\mathrm{O})$ $1720 \mathrm{~cm}^{-1}$. X-ray quality crystals were obtained by slow cooling of the filtrate at $-20{ }^{\circ} \mathrm{C}$. The crystals that were suitable for X-ray diffraction studies contained $\mathbf{6}$ and 7. Slow evaporation of an MeCN solution of $\mathbf{6}$ at $-20{ }^{\circ} \mathrm{C}$ yielded green plates of $\mathbf{6}$, which also co-crystallized with 7 with $\mathrm{MeCN}$ and water molecules.

For the preparation of compound $\mathbf{8}$ (see below), $\mathbf{6}$ was synthesized as follows: $\left[\mathrm{Mn}(\mathrm{dtbsalpn})\left(\mathrm{H}_{2} \mathrm{O}\right)_{2}\right] \mathrm{PF}_{6}(200 \mathrm{mg}, 0.27 \mathrm{mmol})$ was dissolved in distilled $\mathrm{MeCN}(35 \mathrm{~mL})$ and placed in a dropping funnel. This solution was added slowly to a mixture of $\mathrm{H}_{3} \mathrm{DCBI}$ $(48.3 \mathrm{mg}, 0.31 \mathrm{mmol})$ in $\mathrm{MeCN}(15 \mathrm{~mL})$ and $1.0 \mathrm{M} \mathrm{TBAOH}$ $(0.15 \mathrm{~mL})$ under stirring over a period of $3 \mathrm{~h}$. The mixture was stirred further at room temperature for another $3 \mathrm{~h}$. An undissolved white solid was removed by filtration. The resulting greenish brown filtrate was collected and the solvent then removed under vacuum, leaving a dark green solid. A minimal amount of $\mathrm{MeCN}$ was added, and this slurry was stored at $-20{ }^{\circ} \mathrm{C}$ overnight. A greenish white solid was filtered and discarded. The remaining filtrate was left at room temperature for slow evaporation, yielding the desired dark green product, $\mathrm{Mn}$ (dtbsalpn) $\left(\mathrm{H}_{2} \mathrm{DCBI}\right)(70 \%$ yield). Analysis: found (calc): C, 63.5 (63.8); H, 7.3 (7.2); N, 7.9 (7.8).

\section{$\left[\mathrm{Mn}(\mathrm{IV})(\mathrm{dtbsalpn})\left(\mathrm{H}_{2} \mathrm{DCBI}\right)\right]\left(\mathrm{PF}_{6}\right)(\mathbf{8})$}

Complex $6(100 \mathrm{mg})$ was dissolved in $\mathrm{CH}_{2} \mathrm{Cl}_{2}(10 \mathrm{~mL})$ containing $0.10 \mathrm{M} \mathrm{TBAPF} \mathrm{T}_{6}$. This solution was bulk electrolyzed using a platinum mesh electrode maintained at $1.0 \mathrm{~V}$ against a silver wire reference placed directly in the analyte solution. The platinum counter electrode was placed in $0.10 \mathrm{M} \mathrm{TBA}^{+} \mathrm{PF}_{6}{ }^{-}$in $\mathrm{CH}_{2} \mathrm{Cl}_{2}$ and isolated from the bulk solution by a fine glass frit. After passage of $11 \mathrm{C}$, corresponding to one-electron oxidation, the solution had turned a dark green color. The solvent was removed by evaporation under a stream of dry nitrogen. The resulting solid residue was extracted with $\mathrm{Et}_{2} \mathrm{O}$ and filtered. The filtrate was flash evaporated and the dark green solid washed with $\mathrm{Et}_{2} \mathrm{O}$ and hexane to give $\left[\mathrm{Mn}^{\mathrm{IV}}\right.$ (dtbsalpn)( $\left.\left.\mathrm{H}_{2} \mathrm{DCBI}\right)\right]\left(\mathrm{PF}_{6}\right)$. Analysis: found (calc): $\mathrm{C}, 44.70$ (44.74); H, 5.00 (5.10); N, 5.44 (5.49); Mn, 5.32 (5.38). IR: $v(\mathrm{C}=\mathrm{O})$, $1740 ; v(\mathrm{C}=\mathrm{N}), 1600 ; v\left(\mathrm{PF}_{6}\right), 848 \mathrm{~cm}^{-1}$.

\section{Results}

The ligands used in this study and their respective labels are summarized in Fig. 1.

\section{Structural description of complexes}

Compound 1, shown in Fig. 2, has the classical coordination geometry for a $\mathrm{Mn}$ (III) complex containing a strong tetradentate ligand. The [salpn] ${ }^{2-}$ donates two phenolate oxygen atoms and two imine nitrogen atoms to form an equatorial complex. The Jahn-Teller axis of the molecule is oriented perpendicular to this plane, with an axially bound carboxylate of $\mathrm{H}_{2} \mathrm{DCBI}$ and a bound DMF [Mn1-O5, 2.291(2) $⿱$; Mn1-O7, 2.242(2) $\AA$, respectively]. This demonstrates that the neutral imidazole is not a sufficiently strong donor to compete with solvent for the six coordination sites. In the $\mathrm{H}_{2} \mathrm{DCBI}^{-}$ligand the carboxylate oxygen anti to the imidazole nitrogen is ligated to $\mathrm{Mn}$. 
The structure of $\mathrm{Mn}(\mathrm{III})(\mathrm{salpn})(\mathrm{HMeDCBI}) \quad$ (2, Fig. 3) includes a bidentate carboxyimidazole coordination motif. The [salpn] ${ }^{2-}$ must reorganize from the equatorial conformation seen in Fig. 1 to a $\beta$-cis configuration that has been described previously $[41,46]$. The bidentate $[\mathrm{HMeDCBI}]^{-}$may be viewed as having a deprotonated and coordinated carboxylate (Mn1-O3, $2.162 \AA$ ) that is hydrogen bonded to the C-5 exo carboxylic acid of the ligand (O5-O6). The formally neutral imidazole binds to $\mathrm{Mn}$ at a much longer distance (Mn1N3, $2.245 \AA$ ). The expected Jahn-Teller axis is predicted to include this weak Mn-N bond. The imine $\mathrm{N}$ trans to

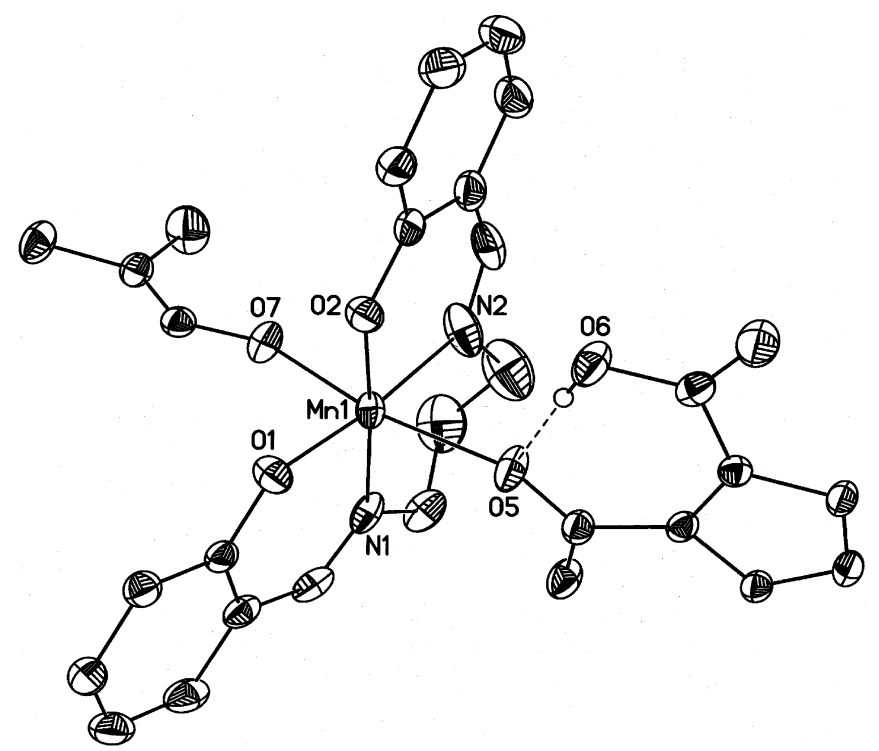

Fig. 2 ORTEP diagram of $\mathrm{Mn}($ salpn $) \mathrm{H}_{2} \mathrm{DCBI}(\mathrm{DMF})$ (1) with ellipsoids $50 \%$ probability

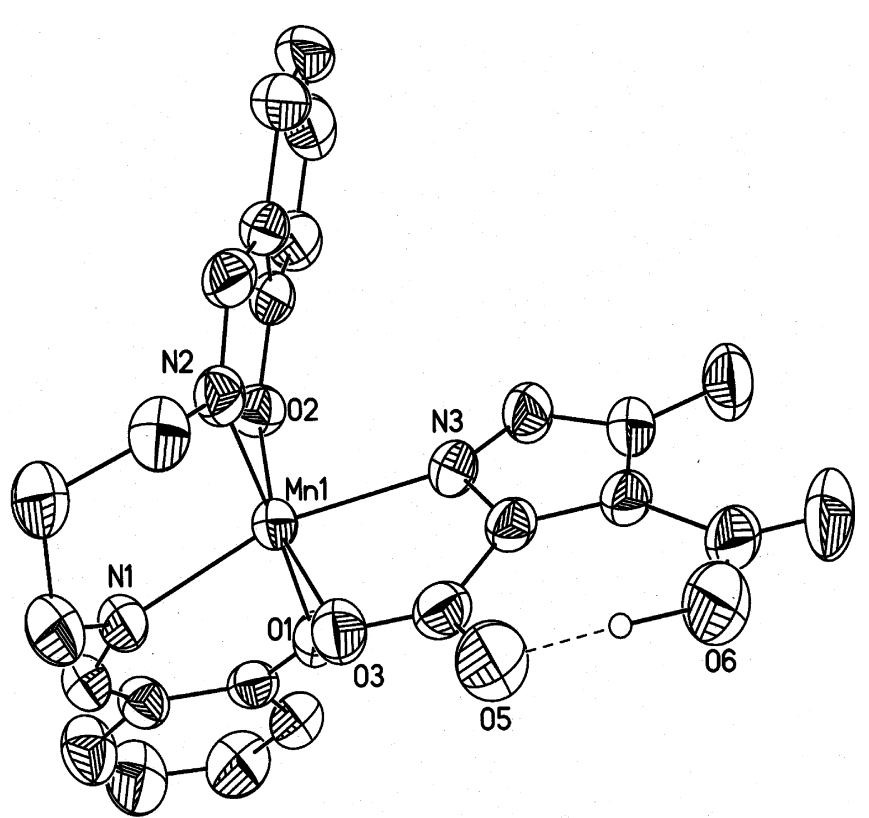

Fig. 3 ORTEP diagram of $\mathrm{Mn}(\mathrm{III})($ salpn)HMeDCBI (2) with ellipsoids $50 \%$ probability the imidazole is in fact bound at a longer distance (Mn$\mathrm{N} 1,2.111 \AA$ ) than the other imine nitrogen of this complex (Mn-N2, $1.995 \AA$ ) or either imine nitrogen of $\mathbf{1}$ (Mn-N2, $2.029 \AA$; Mn-N3, $2.037 \AA$ ).

Compound 6, $\mathrm{Mn}(\mathrm{III})(\mathrm{dtbsalpn})\left(\mathrm{H}_{2} \mathrm{DCBI}\right)$, differs from 2 in subtle, yet significant, ways. The carboxyimidazole is not alkylated; therefore the protons may migrate between heteroatoms. This allows for the negative charge to reorient within the ligand. The strong hydrogen bond between $\mathrm{O} 12$ and $\mathrm{O} 14$ begins to deprotonate the imidazole. As shown in Fig. 4 and Table 2, this hydrogen bond significantly strengthens the Mn3-N9 bond, which decreases to 2.159 A. Furthermore, the $\mathrm{H}$-bond network within the ligand (N10-O13 and O14O12) places more positive charge on the carboxyl group, making the $\mathrm{O} 11$ atom behave as a neutral carbonyl donor. The weakening of the carboxyl interaction is reflected in the long Mn-O11 distance (2.265 $\AA$ ). The most significant consequence is the switching of the Jahn-Teller axis orientation in $\mathbf{6}$ compared to $\mathbf{2}$. In $\mathbf{2}$, the equatorial plane includes the carboxylate oxygen of HMeDCBI, whereas the imidazole nitrogen fills this position in 6. The rotation of the Jahn-Teller axis profoundly influences the relative orientation of the magnetic $\left(\mathrm{d}_{z 2}\right)$ and non-magnetic $\left(\mathrm{d}_{x 2-y_{2}}\right) \mathrm{e}_{\mathrm{g}}$ type orbitals of the Mn(III) ion. We will discuss the potential impact of this manganese orbital reorientation with respect to the OEC below.

\section{Electrochemical properties of the complexes}

The cyclic voltammogram of the mononuclear $\mathrm{Mn}^{\mathrm{III}}$ complex 3 shows a single reversible redox couple at

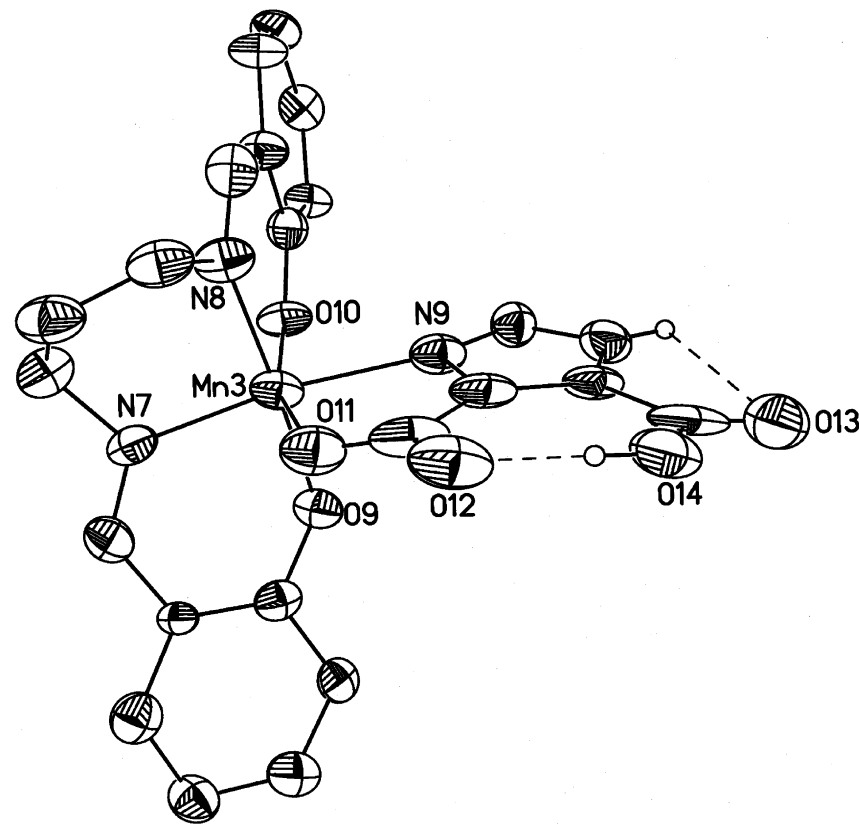

Fig. 4 ORTEP diagram of $\mathrm{Mn}(\mathrm{dtsalpn}) \mathrm{H}_{2} \mathrm{DCBI}(\mathbf{6})$ with ellipsoids $50 \%$ probability 
$E_{1 / 2}=600 \mathrm{mV}$ (vs. $\mathrm{Ag} / \mathrm{Ag}$ wire) and the corresponding $\mathrm{Mn}^{\mathrm{III}}$ complex 6 exhibits an $E_{1 / 2}=690 \mathrm{mV}$ (vs. $\mathrm{Ag} / \mathrm{Ag}$ wire) in dichloromethane. While $\mathbf{3}$ also has a proton hydrogen-bonded to the carboxylate groups of the ligated $(\mathrm{HMeDCBI})$ ligand, attempts to deprotonate it were not successful, even with bases as strong as benzenesulfonamide ( $\left.\mathrm{p} K_{\mathrm{a}}=24.6\right)$ [47]. This suggests that the mononuclear dicarboxyimidazole complexes are substantially less acidic than their dinuclear dicarboxyimidazole-bridged counterparts [46]. While we expect that the $\mathrm{Mn}^{\mathrm{IV}}$ monomer $\mathbf{4}$ should be more acidic, attempts at deprotonation of this complex led to decomposition.

Spectroscopic and magnetic characterization of complexes

The UV-visible spectra of $\mathbf{2}$ and $\mathbf{4}$ were recorded in acetonitrile (Fig. 5). The visible spectrum of the $\mathrm{Mn}^{\text {III }}$ complex 3 possesses a shoulder around $400 \mathrm{~nm}$ and is consistent with $\mathrm{Mn}^{\mathrm{III}}(\mathrm{salpn})$-derived complexes. The oxidized $\mathrm{Mn}^{\text {IV }}$ complex 4 exhibits two shoulders at 350 and $420 \mathrm{~nm}$ and a peak at $710 \mathrm{~nm}$. The band in the visible region is consistent with other high-potential $\mathrm{Mn}(\mathrm{IV})$ complexes having the salpn ligand, and is often assigned as a phenolato-to-metal charge transfer band [48]. The moderately intense bands observed in the nearUV region are due to the overlap of the transition of the azomethine with the charge transfer band from phenolic oxygen to the vacant $\mathrm{d}$ orbital of the Mn(IV).

The X-band EPR spectra of $\mathbf{4}$ and $\mathbf{8}$ are of particular interest (Fig. 6). Compound $\mathbf{4}$ shows a broad signal around $g=4.4$ which follows Curie law behavior. A small signal around $g=2$ can also be observed. The corresponding $\mathrm{Mn}^{\mathrm{IV}}$ monomer $\mathbf{8}$ possesses a broad signal at slightly lower field $(g=5.5)$ than that of 4 . The EPR spectra of $\mathrm{Mn}$ (IV) complexes are highly dependent upon the zero-field splitting parameter, $D$. When $2 D \gg h v$ (the applied microwave frequency), two dominant signals occur with the low-field resonance $(g=4-6)$

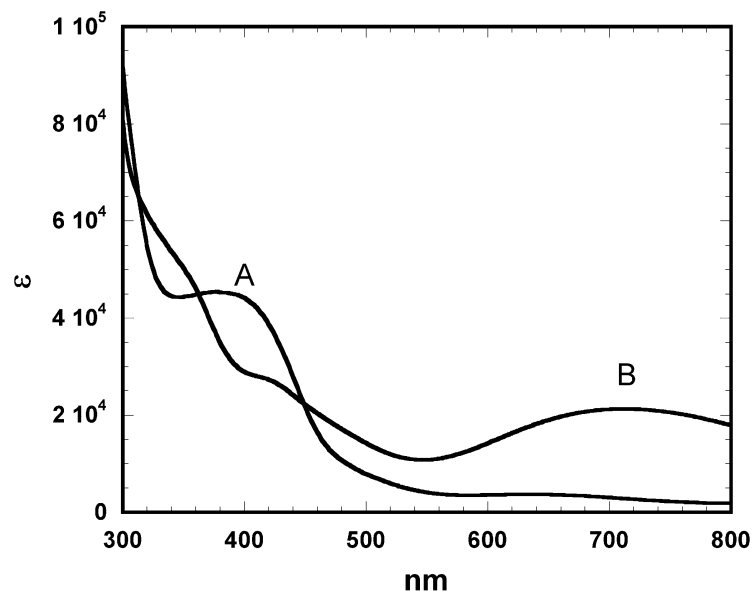

Fig. 5 UV-visible spectra of $2(A)$ and $4(B)$ in acetonitrile being strong and the $g=2$ resonance significantly weaker. The systems become more complex when $2 D \ll h v$. Both compounds $\mathbf{4}$ and $\mathbf{8}$ have $2 D \gg h v$, as seen in Fig. 4. The ratio $E / D$ provides a measure of the axiality for the complex. Perfectly axial spectra will have a $g \approx 4$ resonance whereas the $g$ value will increase with a rhombic electronic distortion. Clearly $\mathbf{8}$ exhibits a significant rhombic distortion that is not observed for $\mathbf{4}$.

Magnetic properties of $\mathrm{Mn}(\mathrm{III})(\mathrm{dtsalpn})(\mathrm{HMeDCBI})(3)$ and $[\mathrm{Mn}(\mathrm{IV})(\mathrm{dtsalpn})(\mathrm{HMeDCBI})] \mathrm{PF}_{6}(\mathbf{4})$

The effective magnetic moment $\left(\mu_{\text {eff }}\right)$ of the mononuclear $\mathrm{Mn}^{\mathrm{III}}$ complex 3 is $\sim 4.9 \mathrm{BM}$ at room temperature, consistent with a high-spin $\mathrm{d}^{4}$ electronic configuration. The $\chi T$ vs. $T$ plot of $\mathbf{3}$ is shown in Fig. 7A, where it is observed that a marked decrease in $\chi T$ occurs below a temperature of $\sim 30 \mathrm{~K}$. The straight line through the data points represents the best fit of Eq. 1 to these data, where $\chi_{\text {ave }}$ is the spatially averaged value of the molar magnetic susceptibility that includes an isotropic $g$ factor and single-ion anisotropy parameter $D$. The principle components of the susceptibility tensor, $\chi_{\|}$and $\chi_{\perp}$, were calculated using the Van Vleck expression, and the second-order perturbation treatment for $\chi_{\text {ave }}$ is valid in the limit $D>>g \beta H$ :

$\chi_{\text {ave }} T=\left(\chi_{\|} / 3+2 \chi_{\perp} / 3\right) T$

The fitting procedure yielded best fit parameters of $g=1.99$ and $\left|D_{\mathrm{Mn}(\mathrm{III})}\right|=4.8 \mathrm{~cm}^{-1}$ for 3. Although it is virtually impossible to determine the sign of $D$ from powder susceptibility data, spin-orbit mixing of ligand field excited states into the ground state usually results in a negative value for $D_{\mathrm{Mn}(\mathrm{III})}$ [49]. The $D_{\mathrm{Mn}(\mathrm{III})}$ value for 3 that we report here is very similar to values previously

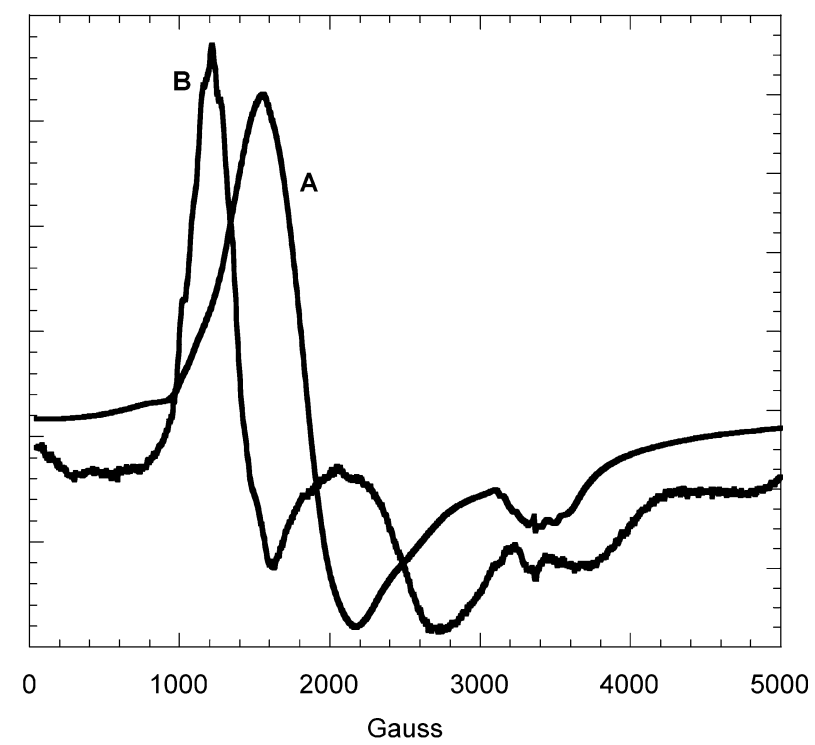

Fig. 6 The X-band EPR spectrum of $\mathbf{4}(A)$ and $8(B)$ at $77 \mathrm{~K}$ in dichloromethane 

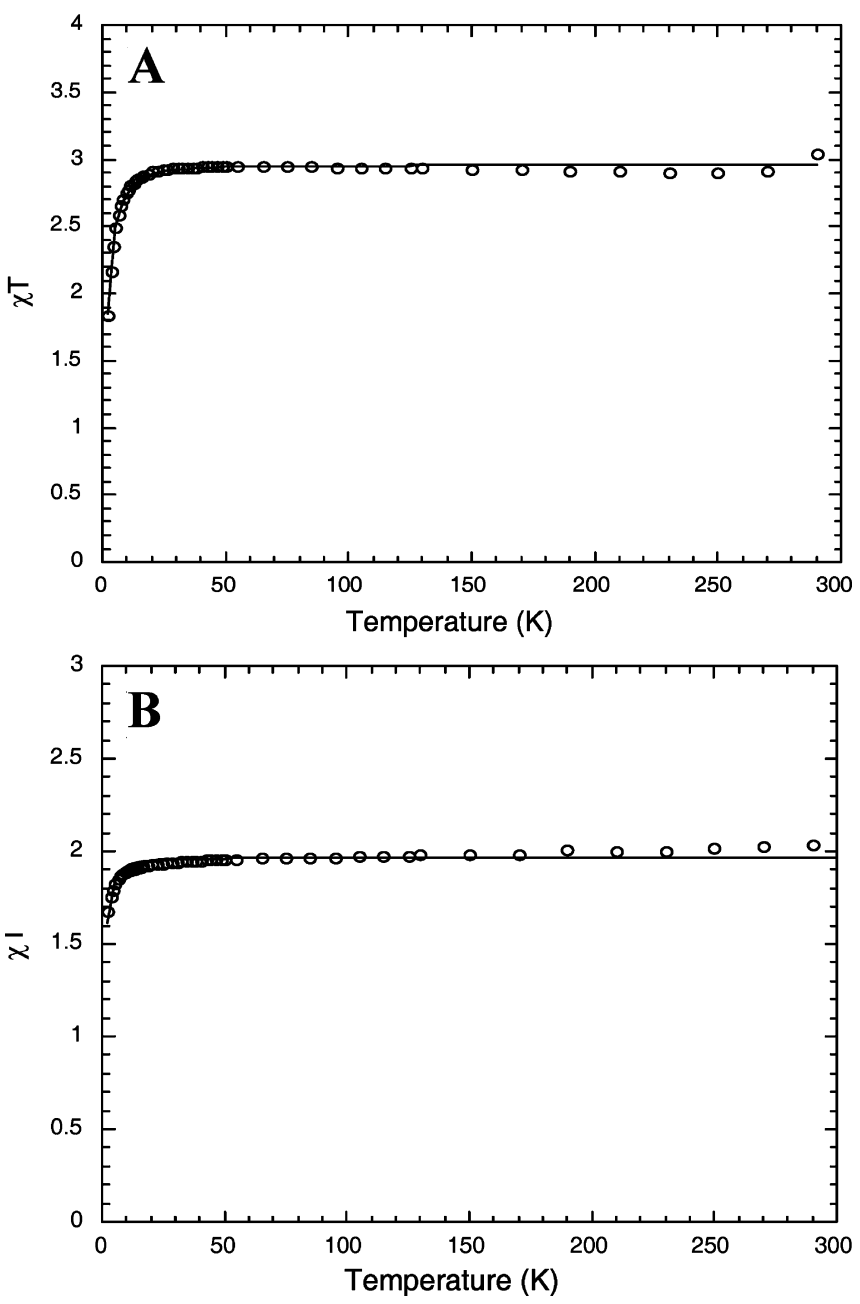

Fig. 7 Temperature dependence magnetic susceptibility plots $(\chi T$ vs. $T$ ) for 3 (A) and 4 (B). Fits to the data are given as a solid line

reported for other $\mathrm{Mn}$ (III) complexes [50]. Similarly, the mononuclear $\mathrm{Mn}^{\mathrm{IV}}$ complex 4 has a magnetic moment of $\sim 4 \mathrm{BM}$ in the high-temperature limit, as expected for an octahedral $\mathrm{d}^{3}$ electronic configuration. The $\chi T$ vs. $T$ plot for $\mathbf{4}$ is shown in Fig. 7B, where it is observed that a marked decrease in $\chi T$ occurs below a temperature of $\sim 30 \mathrm{~K}$ in a manner similar to that observed for 3 . The straight line through the data points represents the best fit of Eq. 1 to these data, and the fitting procedure yielded best fit parameters of $g=2.05$ and $\left|D_{\mathrm{Mn}(\mathrm{IV}}\right|=2.9 \mathrm{~cm}^{-1}$ for 3 . This is an appreciable value for a $\mathrm{d}^{3}$ ion which does not possess first-order angular momentum in $O_{h}$ symmetry.

\section{Discussion}

The $\mathrm{S}_{2}$ state $g=2$ multiline and $g=4.1$ EPR signals

It has been known for many years that the $S_{2}$ state of the OEC is EPR active. Dismukes and Siderer [4] discovered a multiline signal that is centered at $g \approx 2.0$ and Casey and Sauer [51] later reported a signal that appeared around $g=4.1$. Often, samples can be obtained that contain centers that show either of these two signals [51, $52,53]$. It is clear that the $g=2$ multiline signal has as its origin a multinuclear manganese cluster that has a ground state $S=1 / 2$ spin. The $g=4.1$ signal has been much more controversial, both in its preparation and the interpretation of its electronic structure. The addition of small molecules such as ammonia can induce the formation of the $g=4$ signal [20]. It has been reported that low-temperature illumination can trap a $g=4.1$ signal [30] and/or that stray IR radiation can induce the appearance of this low-field feature [23, 24, 25, 26, 27, $28,29]$. Originally, the $g=4.1$ signal was thought to be an excited state of the manganese cluster [54] or a monomeric $\mathrm{Mn}(\mathrm{IV})$ that was in close proximity to the Mn cluster [55]. Both of these cases invoked an $S=3 / 2$ spin system as the signal's origin. Subsequent studies using oriented samples treated with ammonia demonstrated that, for the $g=4$ signal generated under these conditions, the center responsible for the signal was multinuclear [56]. Furthermore, reports that the $g=4.1$ signal is actually due to an $S=5 / 2$ [18] or, when treated with NO, $S=7 / 2$ [57] spin system have been presented. Through these studies, it becomes apparent that there may be several different forms of the cluster that can give a $g=4.1$ signal and that the structures of these centers are perturbed either in large extent or in a small way from the form that gives the $g=2$ multiline signal.

\section{Impact of H-bonding on the OEC}

One possibility for a minor structural perturbation to the system is a trapped protonation state of the cluster. An early explanation for the appearance of the $g=4$ signal during low-temperature illumination of reaction centers suggested that at $120 \mathrm{~K}$ the enzyme was unable to release a proton from the active center; however, upon warming the sample, the proton became mobile and the $g=2$ multiline signal reappeared [58, 59]. While this model has been present for many years, appropriate model compounds that might test this hypothesis and explain on a molecular level how protons can perturb the magnetic behavior of such a system are rare.

We showed that $\mu_{2}$-oxo bridges in Mn(IV) dimers can be protonated $[48,60]$. This protonation reaction leads to an increase in the Mn-Mn distances by about $0.1 \AA$ and dramatically reduces the magnetic exchange interaction. In these cases, an unprotonated dimer had a Heisenberg exchange constant, $J$, on the order of $-120 \mathrm{~cm}^{-1}$, while for the singly protonated dimer $J$ was reduced by half $\left(J \cong 60 \mathrm{~cm}^{-1}\right)$. Finally, the exchange coupling in the diprotonated dimer was reduced by another $50 \mathrm{~cm}^{-1}\left(J=-10 \mathrm{~cm}^{-1}\right)$. These studies showed that the magnitude of the antiferromagnetic coupling could be significantly perturbed if a ligand bridging to manganese ions was protonated. However, there has been little work to address the structural and potential 
magnetic exchange interactions of a manganese system in which a non-bridging ligand is protonated.

The compounds presented in this report provide the first example of how perturbations to H-bonding motifs of non-bridging ligands can cause significant structural rearrangements in manganese compounds. These structural changes have a direct impact on the magnetic orbitals of manganese compounds and could lead to marked perturbations in magnetic exchange coupling between metals. Six-coordinate Mn(III) complexes are subject to a Jahn-Teller distortion that results from the energy lowering that occurs by breaking the degeneracy of the partially filled $\mathrm{e}_{\mathrm{g}}$ orbitals. Usually, this distortion manifests itself as a tetragonal elongation, as is observed in compound 1. Axial distances that are on the order of $0.2-0.3 \AA$ longer than the chemically equivalent bond type in the equatorial plane are typical. In most cases, the elongation occurs in a way that positions the weakest donors on the Jahn-Teller axis. The resultant filling of the $\mathrm{d}$ orbitals places the empty $\mathrm{d}_{x 2-y 2}$ orbital at highest energy. Since $\mathrm{Mn}(\mathrm{III})$ ions in a biological environment are always high spin, the $\mathrm{d}_{z 2}$ orbital is magnetic (that is, half-filled) while the $\mathrm{d}_{x 2-y 2}$ orbital is non-magnetic (unfilled). Thus, the orbital that forms $\sigma$ interactions with the strong equatorial ligands is not a magnetic orbital.

The DCBI ligands can bind to metals in either monodentate or bidentate fashion. In compound $\mathbf{1}$, the monodentate ligand form occurs and the weak carbonyltype oxygen bond (similar in strength to that expected for the carbonyl of DMF) is located on the Jahn-Teller axis. In the bidentate ligand-binding mode, exemplified by compounds $\mathbf{2}$ and $\mathbf{6}$, the strong tetragonal environment presented by the salpn(2-) ligand is disrupted. In $\mathbf{2}$, as shown in Fig. 3, the carboxylate oxygen atom $\mathrm{O} 3$ is trans to the phenolate donor, $\mathrm{O} 2$, and the imidazole nitrogen, N3, is trans to the imine nitrogen, N1. The imidazole donor is strictly neutral due to the alkylation of the second imidazole ring nitrogen, N4. The carboxylate group containing the metal binding atom, O3, forms a weak hydrogen bond (using oxygen atom O5) with the carboxylic acid group (using oxygen atom $\mathrm{O} 6$ ). Thus, the carboxylate atom $\mathrm{O} 3$ carries residual negative charge that binds the $\mathrm{Mn}(\mathrm{III})$. The result is that the tetragonal plane is defined by atoms $\mathrm{O} 1$ and $\mathrm{O} 2$ (phenolate), O3 (carboxylate), and N2 (imine) and the resulting Jahn-Teller elongation occurs along the N1-Mn1-N3 axis.

In contrast, compound 6, shown in Fig. 4, contains the ligand with an unmethylated imidazole ring. In this case, the imidazole nitrogen atom N10 forms a hydrogen bond with oxygen atom $\mathrm{O} 13$ of the adjacent carboxyl group. This hydrogen bond decreases the negative charge on the carboxyl group and increases the negative charge on the imidazole group. Therefore, N9 carries greater negative charge than N3 of compound 2. Similarly, O14 carries less negative charge than O6 of compound 2. Oxygen atom $\mathrm{O} 12$ in $\mathbf{6}$ is forced to accept more positive charge from this hydrogen bond, which lowers the overall negative charge on the carboxylate moiety.
This causes $\mathrm{O} 11$ to be a poorer ligand to the Mn3 atom. The increased donor capability of N9 in conjunction with the decreased donor capability of $\mathrm{O} 11$ cause a rearrangement of the Jahn-Teller axis. The strong equatorial plane is formed by $\mathrm{N} 7$ and $\mathrm{N} 8$ (imine), O9 (phenolate), and N9 (imidazole), whereas the elongated axis contains $\mathrm{O} 10$ (phenolate) and O11 (carboxylic acid). This is a remarkable transformation because an imidazole nitrogen replaces a negatively charge phenolate oxygen in the tetragonal plane. The new orientation of the $\mathrm{d}_{x 2-y 2}$ and $\mathrm{d}_{z 2}$ orbitals corresponds to the [N7, N8, O9, N9] plane and the O10-Mn3-O11 axis, respectively. A summary of the H-bonding schemes for these compounds is provided in Fig. 8.

A related perturbation of Jahn-Teller axes by deprotonation has been reported by Corbella et al. [61]. These authors showed that conversion of a bound hydroxide ion to an aqua ligand on a dinuclear $\mathrm{Mn}$ (III) complex dramatically perturbed the magnetic exchange interactions. Therefore, H-bonding changes, as seen in $\mathbf{2}$ and $\mathbf{6}$, would be expected to cause significant magnetic perturbations in dimers or clusters as well as simple monomers.

One can imagine a scenario in which oxidation of the manganese cluster in the OEC occurs without the release of a proton from a ligand coordinated to an adjacent $\mathrm{Mn}$ (III) ion [in $\mathrm{S}_{2}$, the metal oxidation states are $\left.\mathrm{Mn}(\mathrm{III}) \mathrm{Mn}(\mathrm{IV})_{3}\right]$. While the proton is trapped, the $\mathrm{Mn}$ (III) could have the Jahn-Teller axis oriented in such a manner that the measured Mn(III)-Mn(UIV) antiferromagnetic exchange $\left(J=J_{\text {af }}+J_{\mathrm{f}}\right)$ is severely reduced either bei decreasing $J_{\text {af }}$ or by turning on new ferromagnetic exchange pathways $\left(J_{\mathrm{f}}\right)$. Such a cluster configuration could give a large ground-state spin $(S=5 / 2$ or $7 / 2$ ) and a $g=4.1$ signal. Upon warming, and proton movement, the ligands on the Mn(III) could rearrange, causing a realignment of the magnetic orbitals in such a

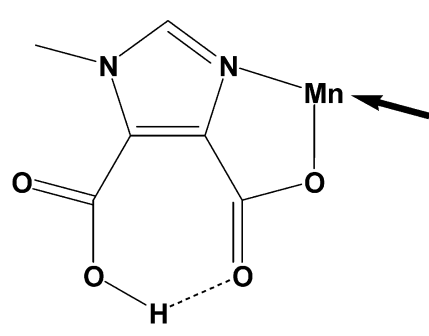

A

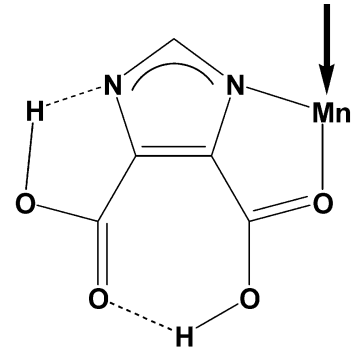

B
Fig. 8A, B Hydrogen bonding in compounds 2 and 6. A Complex $\mathbf{2}$ in which the methyl group of the ligand does not allow hydrogen bonding to the uncoordinated imidazole nitrogen atom. B A strong hydrogen bond is formed between the uncoordinated imidazole nitrogen and uncoordinated carboxylate oxygen atoms. The strength of the bound carboxylate and imidazole nitrogens invert between $\mathbf{2}$ (strong oxygen, weak nitrogen) and $\mathbf{6}$ (strong nitrogen and weak oxygen). The direction of the Jahn-Teller axis in the molecules is shown with the dark arrow 
way as to facilitate antiferromagnetic exchange, giving an $S=1 / 2$ ground state and a $g=2$ multiline signal. EXAFS measurements of the $\mathrm{S}_{2}$ state with the cluster in the $g=2$ and $g=4$ configurations indicate that the structure is invariant [23]. The model presented above would also be expected to cause minor, EXAFS indescernable perturbations to the overall cluster structure.

The structural perturbations observed with these manganese compounds are related to the push-pull mechanisms of H-bonding in heme proteins. Examination of proteins such as hemoglobin or chloroperoxidases reveals that $\mathrm{H}$-bonding of residues to the distal imidazole can dramatically alter the electronic structure of the heme unit and the reactivity of the compound. One can imagine an analogous $\mathrm{H}$-bonding scheme in photosystem II whereby release of protons from the vicinity of the manganese center not only impacts the energetics of water oxidation chemistry (by causing charge neutral transitions), but also directly influences the structural and electronic properties of the cluster itself. The studies reported herein now provide the first documented case where the simple expedient of altered $\mathrm{H}$-bonding on a non-bridging ligand can be directly traced back to a significant structural rearrangement of the manganese coordination sphere.

Acknowledgements The authors thank the National Institute of Health for funding (GM39406 to V.L.P. and GM-057378 to M.L.K.) and a postdoctoral fellowship (GM17348 to M.T.C).

\section{References}

1. Radmer R, Cheniae G (1997) In: Barber J (ed) Primary processes of photosynthesis. Elsevier, Amsterdam, pp 303-348

2. Pecoraro VL, Hsieh W-Y (2000) In: Sigel A, Sigel H (eds) Metal ions in biological systems, vol 37. Dekker, Basel, pp 429-504

3. Cheniae GM (1980) Methods Enzymol 69:349-363

4. Dismukes GC, Siderer Y (1980) FEBS Lett 121:78-80

5. Keele BB Jr, McCord JM, Fridovich I (1979) J Biol Chem 245:6176-6181

6. Ludwig ML, Metzger AL, Pattridge KA, Stalling WC (1991) J Mol Biol 219:335-358

7. Stalling WC, Pattridge KA, Strong RK, Ludwig ML (1985) J Biol Chem 260:16424-16432

8. Borgstahl GEO, Parge HE, Hickey MJ, Beyer WF, Hallewell RA, Trainer JA (1992) Cell 71:107-118

9. Kanyo ZF, Scolnick LR, Ash DE, Christianson DW (1996) Nature 383:554-557

10. Barynin VV, Grebenko AI (1986) Dokl Akad Nauk SSSR 286:461-464

11. Barynin VV, Vagin AA, Melik-Adamyan, Grebenko AI, Khangulov SV, Popov AN, Andrianova ME, Vainshtein BK (1986) Dokl Akad Nauk SSSR 288:877

12. Debus RJ (1992) Biochim Biophys Acta 1102:269-352

13. Ort DR, Yocum CF (1996) (eds) Oxygenic photosynthesis: the light reactions. Kluwer, Boston

14. Lorigan GA, Britt RD (1994) Biochemistry 33:12072-12076

15. Cole J, Yachandra VK, Guiles RD, Macdermott AE, Britt RD, Dexheimer SL, Sauer K, Klein MP (1987) Biochim Biophys Acta 890:395-398

16. Kim DH, Britt RD, Klein MP, Sauer K (1987) J Am Chem Soc 112:9389-9391
17. Yachandra VK, DeRose VJ, Latimer MJ, Mukerji I, Sauer K, Klein MP (1993) Science 260:675-679

18. Haddy A, Dunham WR, Sands RH, Aasa R (1992) Biochim Biophys Acta 1099:25-34

19. Bennoun P, Joliot A (1969) Biochim Biophys Acta 189:85-94

20. Britt RD, Zimmermann JL, Sauer K, Klein MP (1989) J Am Chem Soc 111:3522-3532

21. Baumgarten M, Philo JS, Dismukes GC (1990) Biochemistry 29:10814-10822

22. De Rose VJ, Latimer MJ, Zimmerman JL, Mukerji I, Yachandra VK, Sauer K, Klein MP (1995) Chem Phys 194:443-459

23. de Paula JC, Beck WF, Brudvig GW (1986) J Am Chem Soc 108:4002-4009

24. Dismukes GC, Siderer Y (1981) Proc Natl Acad Sci USA 78:274-278

25. Hansson O, Andreasson LE, Vangard T (1986) FEBS Lett 195:151-154

26. Beck WF, de Paula JC, Brudvig GW (1986) J Am Chem Soc 108:4018-4022

27. Beck WF, Brudvig GW (1986) Biochemistry 25:6479-6486

28. Beck WF, de Paula JC, Brudvig GW (1985) Biochemistry 24:3035-3043

29. de Paula JC, Innes JB, Brudvig GW (1985) Biochemistry 24:8114-8120

30. Forster V, Junge W (1985) Photochem Photobiol 41:183-190

31. Kirk ML, Chan MK, Armstrong WH, Solomon EI (1992) J Am Chem Soc 114:10432-10440

32. Philouze C, Blondin G, Girerd JJ, Guilhem J, Pascard C, Lexa D (1994) J Am Chem Soc 116:8557-8565

33. Zouni A, Witt HT, Kern J, Fromme P, Kraub N, Saenger W, Orth P (2001) Nature 409:739-743

34. Tang XS, Sivaraja M, Dismukes GC (1993) J Am Chem Soc 115:2382-2389

35. Goldberg J, Haung HB, Kwon YG, Greenwood P, Nairn AC, Kuriyan J (1995) Nature 376:745-753

36. Kanyo ZF, Scolnick LR, Ash DE, Christianson DW (1996) Nature 383:554-557

37. Lehnert R, Seel FZ (1980) Z Anorg Allg Chem 464:187-194

38. Seela JL, Kolting K, Wang RJ, Huffman JC, Christou G, Chang HR, Hendrickson DN (1985) Inorg Chem 24:4454-4456

39. Rypniewski WR, Mangani S, Bruni S, Orioli PL, Casati M, Wilson K (1995) J Mol Biol 251:281-296

40. Trainer JA, Getzoff ED, Beem KM, Richardson JS, Richardson DC (1982) J Mol Biol 160:181-217

41. Caudle MT, Kampf JW, Kirk ML, Rasmussen PG, Pecoraro VL (1997) J Am Chem Soc 119:9297-9298

42. Sheldrick GM (1988) SHELXTL PLUS structure determination programs. Nicolet Instrument, Madison, Wis

43. Ibers JA, Hamilton WC (eds) (1974) International tables for crystallography, vol 4. Knoch Press, Birmingham, UK, entry 55-99-149

44. O'Connell EJ, Parquette J, Yelle WE, Wang W, Rapoport H (1988) Synthesis 10:767-771

45. Larson EJ, Pecoraro VL (1991) J Am Chem Soc 113:3810-3818

46. Rajendiran TM, Kirk ML, Setyawati IA, Caudle MT, Kampf JW, Pecoraro VL (2002) (submitted)

47. Izutsu K (1990) Acid-base dissociation constants in dipolar aprotic solvents. Blackwell, Boston

48. Boldwin MJ, Stemmler TL, Riggs-Gelasco PJ, Kirk ML, Penner-Hahn JE, Pecoraro VL (1994) J Am Chem Soc 116:11349-11356

49. Dugad LB, Behere DV, Marathe VR, Mitra S (1984) Chem Phys Lett 104:353-356

50. Krzystek J, Tesler J, Pardi LA, Goldberg DP, Hoffman BM, Brunel LC (1999) Inorg Chem 38:6121-6129

51. Casey JL, Sauer K (1984) Biochim Biophys Acta 767:21-28

52. Zimmerman JL, Rutherford AW (1984) Biochim Biophys Acta 767:160-167

53. Zimmerman JL, Rutherford AW (1986) Biochim Biophys Acta 25:4609-465

54. Astashkin AV, Kodera Y, Kawamori A (1994) J Magn Reson 105:113-119 
55. Hansson O, Aasa R, Vanngard T (1987) Biophys J 51:825-832

56. Kim DM, Britt RD, Klein MP, Sauer K (1990) J Am Chem Soc 112:9389-9391

57. Sanakis Y, Ioannidis N, Sioros G, Petrouleas V (2001) J Am Chem Soc 123:10766-10767

58. de Paula JC, Beck WF, Miller AF, Wilson RB, Brudvig GW (1987) J Chem Soc Faraday Trans 83:3635-3651
59. Zimmermann JL, Rutherford AW (1986) Biochemistry 25:4609-4615

60. Larson EJ, Riggs-Gelasco PJ, Penner-Hahn JE, Pecoraro VL (1992) J Chem Soc Chem Commun 102-103

61. Corbella M, Costa R, Ribas J, Fries PH, Latour JM, Ohrstrom L, Solans X, Rodriguez (1996) Inorg Chem 35:1857-1865 